\title{
Preparation of Polyelectrolyte Hydrogels and Study Their Controlled Release of Gabapentin
}

\author{
Zaid A Abdulmonaim and Athir M Haddad* \\ Department of Chemistry, University of Basrah, Iraq \\ Received: 阱 March 25, 2018; Published: 畊 April 04, 2018 \\ *Corresponding author: Athir M Haddad, Professor of Polymer Chemistry, Iraq
}

\begin{abstract}
The semi interpenetrating network hydrogels (Z1-Z15) were prepared from different ratio of sodium alginate and linear poly (acrylamide-co-diallyl dimethyl ammonium chloride) then they mixed with the acrylamide and bisacrylamide as cross linking agent and polymerized via redox polymerization to form the semi-IPNs. All prepared semi-IPNs were loaded with three different amounts from Gabapentin as drug model. The swelling characteristics were studied for all semi- IPN hydrogels by determining the swelling ratio (Q).The release of Gabapentin was followed by using U.V. spectroscopy at $(202,201$ and 204) nm at constant temperature $\left(37^{\circ} \mathrm{C}\right)$ in distilled water, simulated gastric fluid (SGF) and simulated intestinal fluid (SIF) respectly. The results of Gabapentin released indicated that all semi-IPNs have the ability to release drug to environment and the amount of Gabapentin released about $50 \%$ during two hours. Higuchi equation was used to determine the Higuchi constant which is very useful to forecasting the amount of Gabapentin released theoretically.
\end{abstract}

Keywords: Polyelectrolyte; Polymer Drug Delivery; Gabapentin

Abbreviations: SGF: Simulated Gastric Fluid; SIF: Simulated Intestinal Fluid; TDDSs: Targets Drug Delivery Systems; CDDSs: Controlled Drug Delivery Systems; SGF: Systems In Simulated Stomach Fluid; SIF: The Simulated Intestinal Fluid; WU: Water Uptake

\section{Introduction}

It is rare to use drugs as pure chemicals alone, it is given as the formula of drug structures such as drug delivery systems. Simple solutions can be developed to form these systems through the use of appropriate additives or excipients to prepare the pharmacological structures. The effectiveness of many of drugs is often designed to working at the site of the therapeutic act, So the method of delivery of medication can have a significant impact on its effectiveness. In many cases conventional medications reach the target location in a small amount while the most amount of the medication is distributed in the whole of the body according to its physicochemical and biochemical properties, so it has developed drug delivery systems that improve the drug's pharmacologic action, reduce side effects and toxicity within the body of the organism $[1,2]$. The transition from simple tablets to tablets of continuous release and the discovery of sophisticated programmable delivery systems led to the delivery of drugs to the target and cells are more accurate [3].

The synergy between chemists, biologists, doctors and biomedical engineers over the past 20 years has led to the development of controlled release technology and has been the best solution for drug delivery systems. It is a type of drug products that is controlled for long periods of time with maintaining drug concentration in the blood Within optimal treatment limits $[4,5]$. It is justified to resort to these systems instead of traditional medication formulas due to the problems in metabolism or absorption of medication when using traditional systems or to improve the treatment itself and the release of the drug must be in the right part of the body and the rates required for treatment $[6,7]$. Controlled drug delivery systems are designed to deliver drugs at 
controlled rates for specified periods of time and release them at required rates in places where treatment is needed. The concept of drug delivery systems can be classified into two categories:

i. Targeting is one of the controlled release systems and works on delivery of the active ingredient only to the required tissue or organs called Targets Drug Delivery Systems (TDDSs). Examples of such systems include the delivery of chemotherapeutic drugs to tumour sites directly without damage to other healthy tissues [8].

ii. The systems that control the rate and speed of release of the active substance are known as Controlled Drug Delivery Systems (CDDSs) [9].

Polymers are the most materials which widely used in controlled release systems because their manufacturing processes are easy and widely used to design drug release systems as well as easy control of their physical and chemical properties during preparation $[10,11]$. There are several types of controlled release formulations [12], the first type is long-range release system(sustained release), It provides effective concentrations for long periods of time and reduces the repetition of taking doses to improve therapeutic compliance and reduce the need for repeated visits to clinics $[13,14]$. The second is Prolonged release system; this system reduces the release of the active ingredient, resulting in a reduction in the toxic effects of this substance and maintenance of the therapeutic activity required for it $[15,16]$.The third is Delayed release; This system delays the release and then release of the active substance without hindrance, for example oral capsules that remain in the stomach intact and break down only at the highest acidic function in the small intestine or colon [17]. The fourth is Repeat action dosage forms; this system is designed to release one dose of medication and for a certain period of time the other dose is released and thus $[16,18]$.

This work consist of preparation of a number of new electrolytic gels polymeric compositions adopted in the composition of the presence of (Acryl Amide, Sodium alginate, linear copolymer (Acryl Amide -co-diallyl dimethyl lammonium chloride) as semi interpenetrating polymer network (semi-IPN) with different

Table 1: The composition used in the preparation of semi-IPNs Z1-Z15.

\begin{tabular}{|c|c|c|c|c|c|c|}
\hline \multirow{2}{*}{ Sample } & \multirow{2}{*}{$\begin{array}{c}\text { P(AAm - co }- \\
\text { DADMAC) }\end{array}$} & Sodium alginate & Drug & \multirow{2}{*}{ AAm g } & \multirow{2}{*}{$\begin{array}{c}\text { Bis AAm g } \\
\text { Ratio Copolymer } \\
\text { and SA: AAm }\end{array}$} \\
\cline { 3 - 6 } & 1 & $\mathbf{g}$ & $\mathbf{g}$ & 2 & 0.2 & $1: 01$ \\
\hline Z1 & 1 & 2 & 0.2 & 3 & 0.3 & $1: 01$ \\
\hline Z2 & 1 & 3 & 0.2 & 4 & 0.4 & $1: 01$ \\
\hline Z3 & 2 & 1 & 0.2 & 3 & 0.3 & $1: 01$ \\
\hline Z5 & 3 & 1 & 0.2 & 4 & 0.2 & $1: 01$ \\
\hline Z6 & 1 & 1 & 0.4 & 2 & 0.3 & $1: 01$ \\
\hline Z7 & 1 & 2 & 0.4 & 3 & & $1: 01$ \\
\hline
\end{tabular}




\begin{tabular}{|c|l|l|l|l|l|l|}
\hline Z9 & 2 & 1 & 0.4 & 3 & 0.3 & 0.4 \\
\hline Z10 & 3 & 1 & 0.4 & 4 & 0.2 & $1: 01$ \\
\hline Z11 & 1 & 1 & 0.6 & 2 & 0.3 & $1: 01$ \\
\hline Z12 & 1 & 2 & 0.6 & 3 & 0.4 & $1: 01$ \\
\hline Z13 & 1 & 3 & 0.6 & 3 & 0.3 & $1: 01$ \\
\hline Z14 & 2 & 1 & 0.6 & 4 & 0.4 & $1: 01$ \\
\hline Z15 & 3 & 1 & 0.6 & 3 & & \\
\hline
\end{tabular}<smiles>[Y]C(C)(C)C(CC(C)(C)C)C1C[N+](C)(C)CC1CC([Y])(C)C(CC(C)(C)C)C(N)=O</smiles>

P(AAm-Co-DADMAC) (Polycation)<smiles>C=CC(N)=O</smiles>

AAm

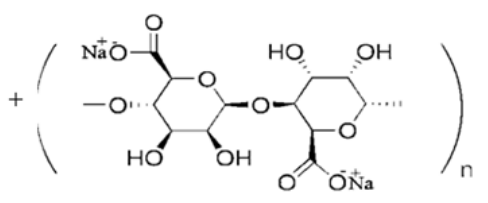

SA

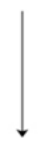

(Polyanion)

Polvelectrolvte Composite<smiles>C=CC(=O)NCNC(=O)C=C</smiles>

BisAAm<smiles>C1CCCCC1</smiles>

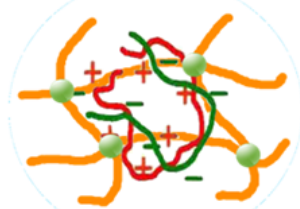

Scheme1: the polymerization reactions for preparation of semi-IPNs;-_poly acrylamide network, —Sodium alginate, —copoly (AAm-Co-DADMAC), •crosslinking poin.

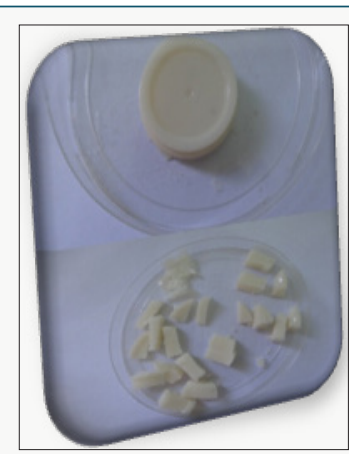

Figure 1: photograph of Z1.

\section{Preparation of The Simulated Stomach Fluid And The} Simulated Intestinal Fluid

The simulated stomach fluid (SGF) and the simulated intestinal fluid were prepared according to the US Encyclopaedia of Drugs [19].

\section{Determination of Standard Calibration Curves For}

\section{Capapentin}

The $\lambda$ max was determined for Gabapentin which showed absorption at (202 nm) in distilled water and (201 nm), (204 nm) in SGF and SIF respectively Then, the standard calibration curves of the drug were determined.

\section{Results and Discussion}

The swelling ratio of the prepared semi-IPN hydrogels was measured as a function of time in distilled water, SFG and SIF at $37^{\circ} \mathrm{C}$. The polymer compositions were weighed with a weight of 0.5 g, during certain periods of time, the samples were removed from the solution and placed on the filter paper to remove the solution from the surface (Interfacial solutions) and weighed to calculating the swelling ratio. The swelling ratio was calculated within 24 hours based on the following equation [20]: 


$$
\% Q=\frac{W s}{W d} \times 100
$$

Whereas; $\mathrm{Q}=$ swelling ratio, $\mathrm{Wd}$, = dry weight of hydrogels and Ws, = the weight of the swollen hydrogels.

The results in Figures 2- 4 indicate that the polymer compositions reach to the swelling equilibrium after 6 hours however the experiment continued for 24 hours. Semi-IPN Z1-
Z15 was loaded with Gabapentin. Copolymers were selected and sodium and acryl amide were selected. Acryl amides are formed by bonding, linear copolymers and sodium alginate. There is a simple ionic interference between the linear copolymer as a weak poly action with sodium nitrite as a strong poly anion to form the polycarbonate structure, which interferes with the formation of the second linear component of the quasi-retina structures prepared.

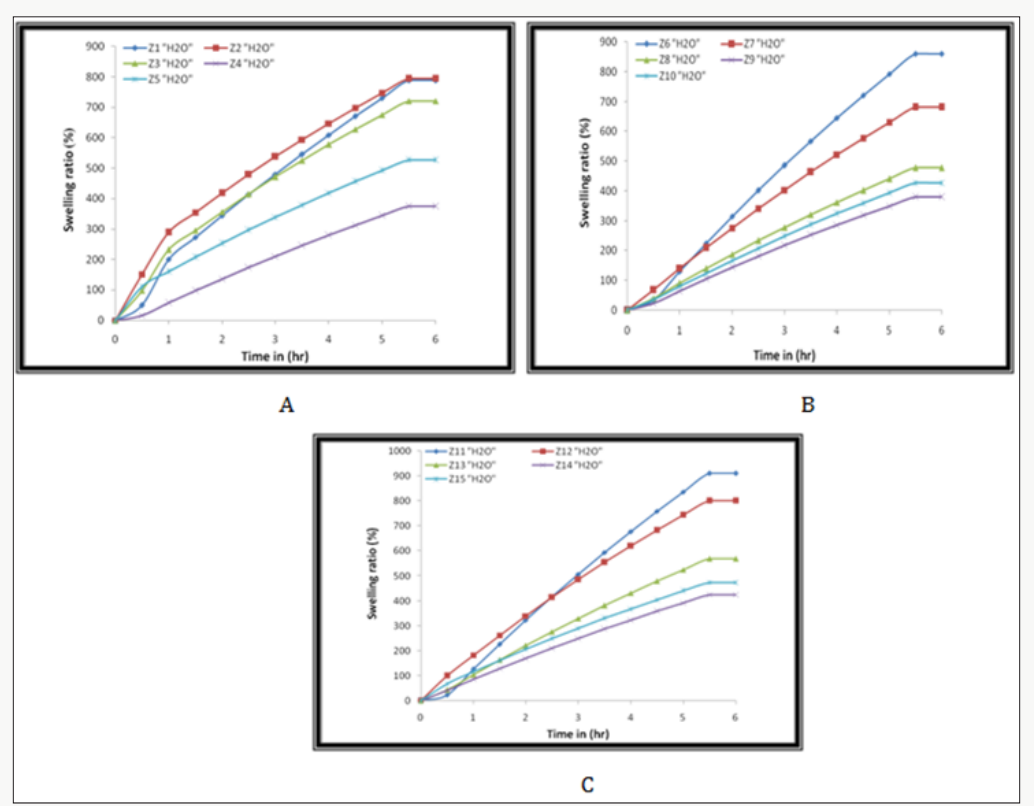

Figure 2: The swelling ratio of Semi-IPN in distilled water; A) Z1-Z5, B) Z6-Z10 and C) Z11-Z15.

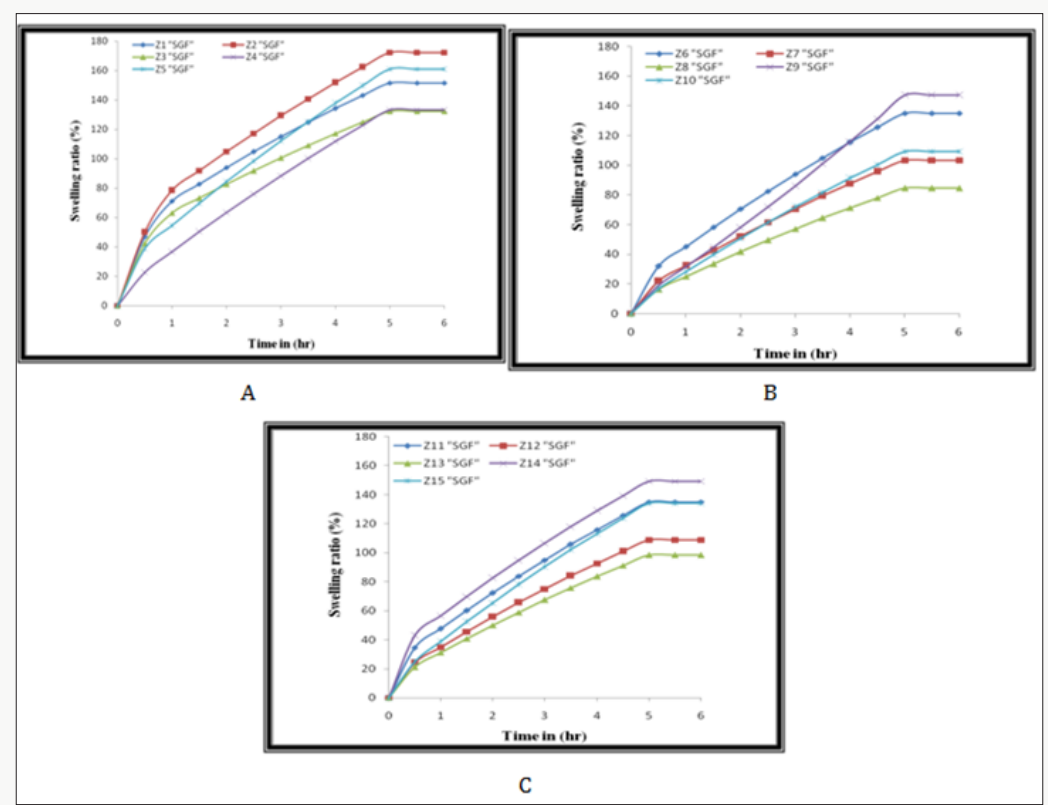

Figure 3: The swelling ratio of Semi-IPN in SGF;A) Z1-Z5 , B) Z6-Z10 and C) Z11-Z15.

(Figures 2-4) were showed high swelling ratio (SR) in both distilled water and SIF due to the presence of carboxylic group anions (- COO-) along the polymer chains of sodium alginate, leading to increased electrical repulsion between the negative charge and then occur a high swelling process for cross linked poly acrylamide network [21-23] as well as the presence of hydrophilic groups such as (-CONH2, -N+R4) in the semi-IPNs of Z1-Z15 compositions, have an important effect on swelling behaviour 
and play an important role in the rate of swelling due to increase hydrogen bonding between polymer networks and water. On the other hand, in the SGF, the semi-IPNs were showed a low swelling ratio due to the protonation of the carboxylate groups in the acid medium lead to coiling of sodium alginate chains around itself and this is enhanced by the non-expansion of the poly acrylamide network structure [21] so the resulting swelling is because of the presence of amide and ammonium groups. In general, there are a number of interactions in the acrylamide network such as ionic interference from polyelectrolyte and hydrogen bonding interference, the amide groups in the poly acrylamide network, work together to form internal hydrogen bonds. These bonds are used to form a stable shell around the ionic groups on both of the polyelectrolyte and then the acrylamide network works to pull the water (water uptake WU) from the environment [24.25]. It was found that the quaternary ammonium groups of copolymers and carboxyl groups in the sodium alginate enhance the change in the swelling ratio in SGF, SIF and distilled water. This can be useful for optimizing the applications of these polymers to the release of drugs or as biomaterials [26].

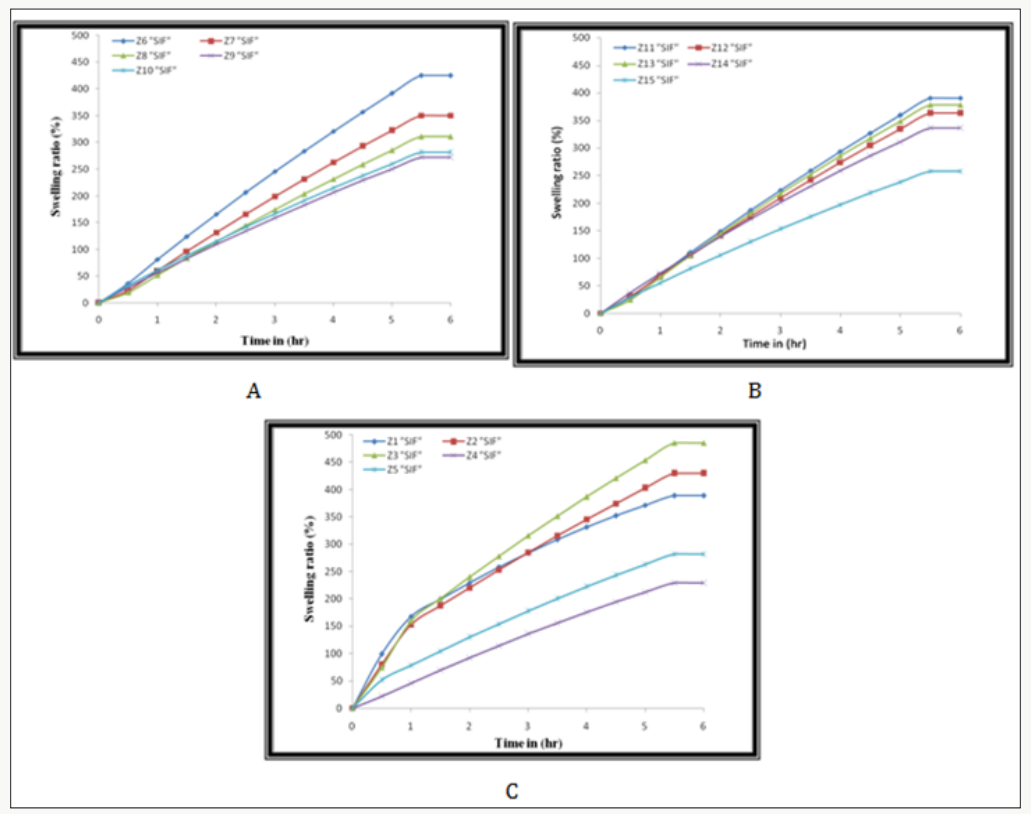

Figure 4: The swelling ratio of Semi-IPN in SGF; A) Z1-Z5, B) Z6-Z10 and C) Z11-Z15.

The external surface morphology of semi-IPNs was studied by the scanning electron microscopy. As in (Figure 5), where the images $A$ and $B$ represent the dry state of the Xerogel structures and swollen gel of Z7 respectively, we observe that they have a smooth compact surface as a result of the completion of the cross linking process of poly Acryl Amide while the swollen state possessed the same fine surface properties and pore sizes ranging from 169.945.48 micrometres.

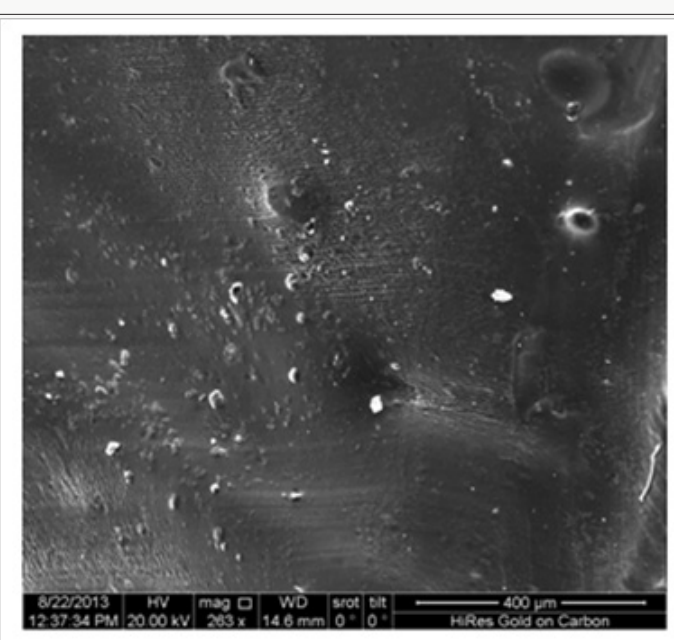

A

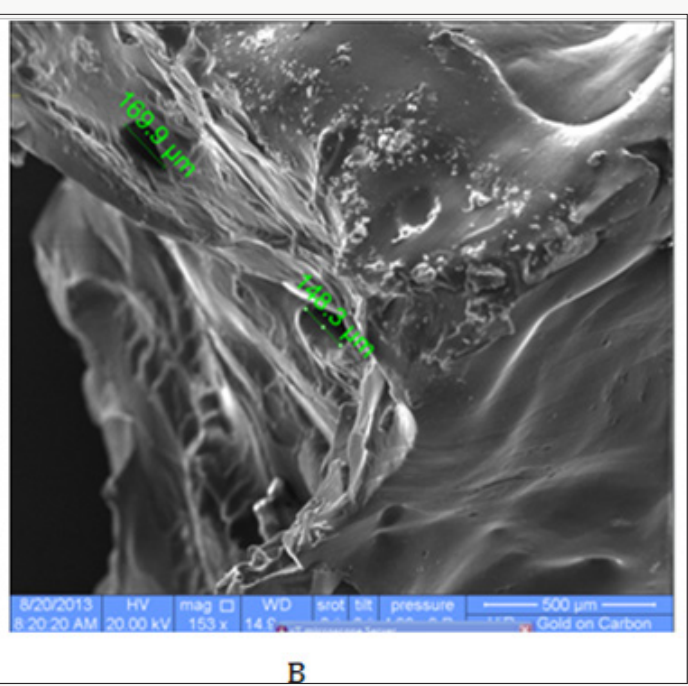

Figure 5: SEM micrograph of A) the xerogel state of Z7 B) swollen state of Z7. 
The \% released of Gabapentin with time was studied at (37 ${ }^{\circ} \mathrm{C}$ ) for six hours, (Figures 6-8) shows the results of this study, the results were showed that there are no significant differences in the rate of release of the drug when immersing these hydrogels in distilled water, SGF and SIF. On the other hand, the prepared semiIPNs were loaded with different amounts of Gabapentin (0.2, 0.4 and $0.6 \mathrm{~g}$ ) and it was found that there is no significant effect of the change in the amount of drug loaded in the prepared hydrogels due to large pore size of the swollen hydrogel leading to the formation of channels within the polymer structures allowing the drug to pass through it to the medium. For this reason, the release of the drug depends on the mechanism of swelling followed by diffusion which is commonly used in gels polymers. This explanation is confirmed by the relatively rapid release of the drug.

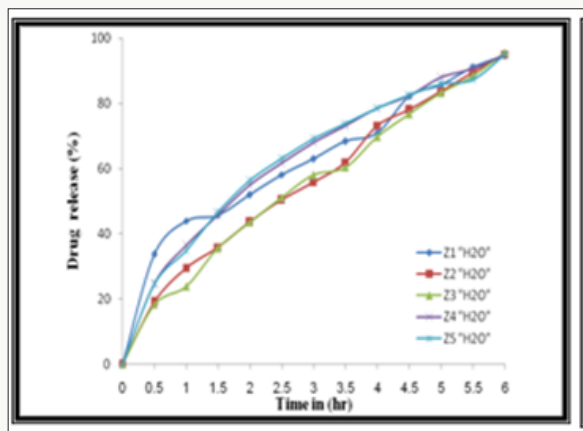

A

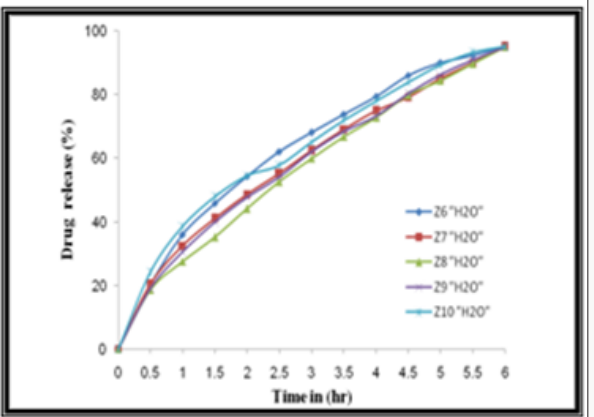

B

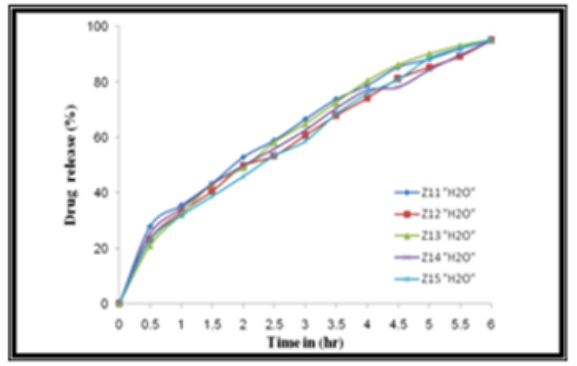

C

Figure 6: The \%Release of Gabapentin from: A) Z1-Z5, B) Z6-Z10 and C) Z11-Z15,in distilled water.

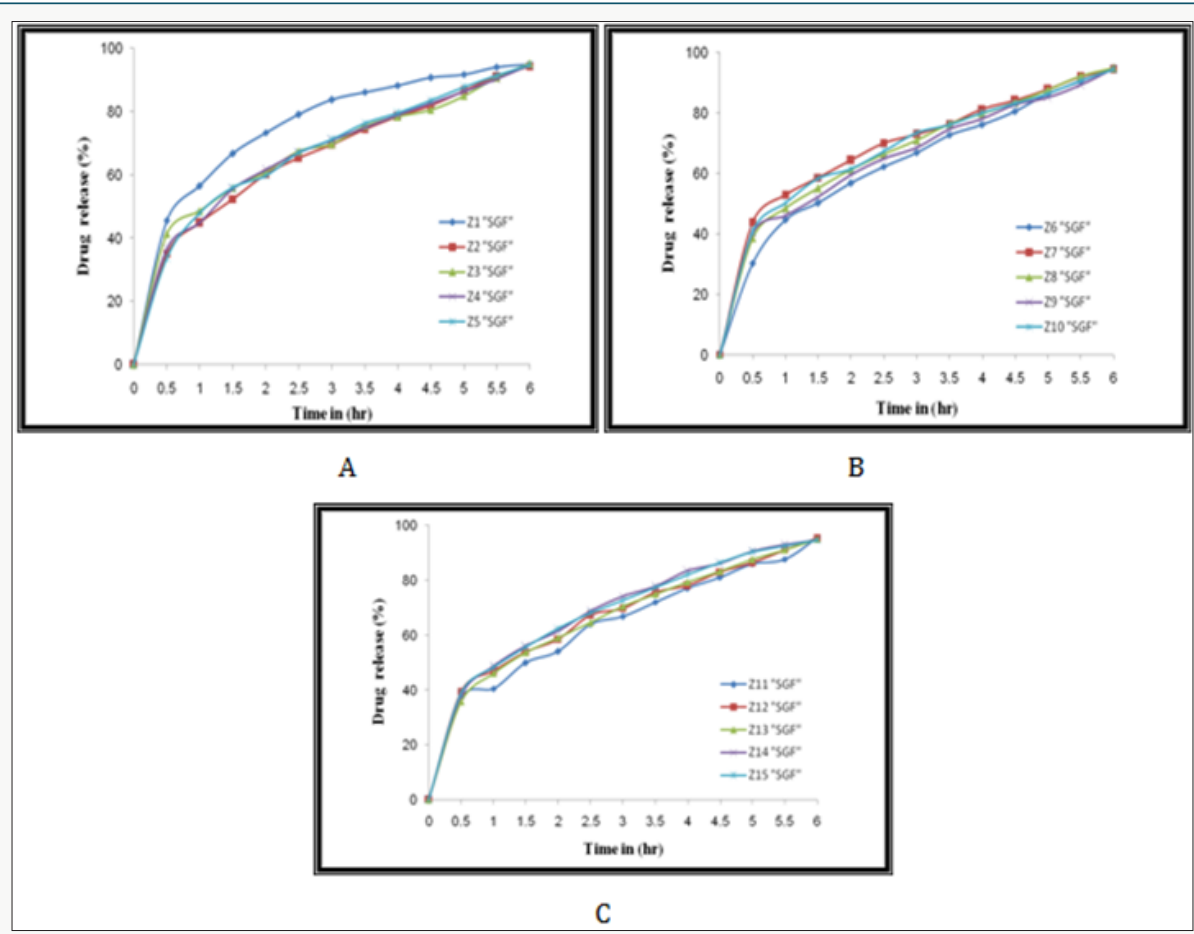

Figure 7: The \%Release of Gabapentin from: A) Z1-Z5, B) Z6-Z10 and C) Z11-Z15, in SGF. 


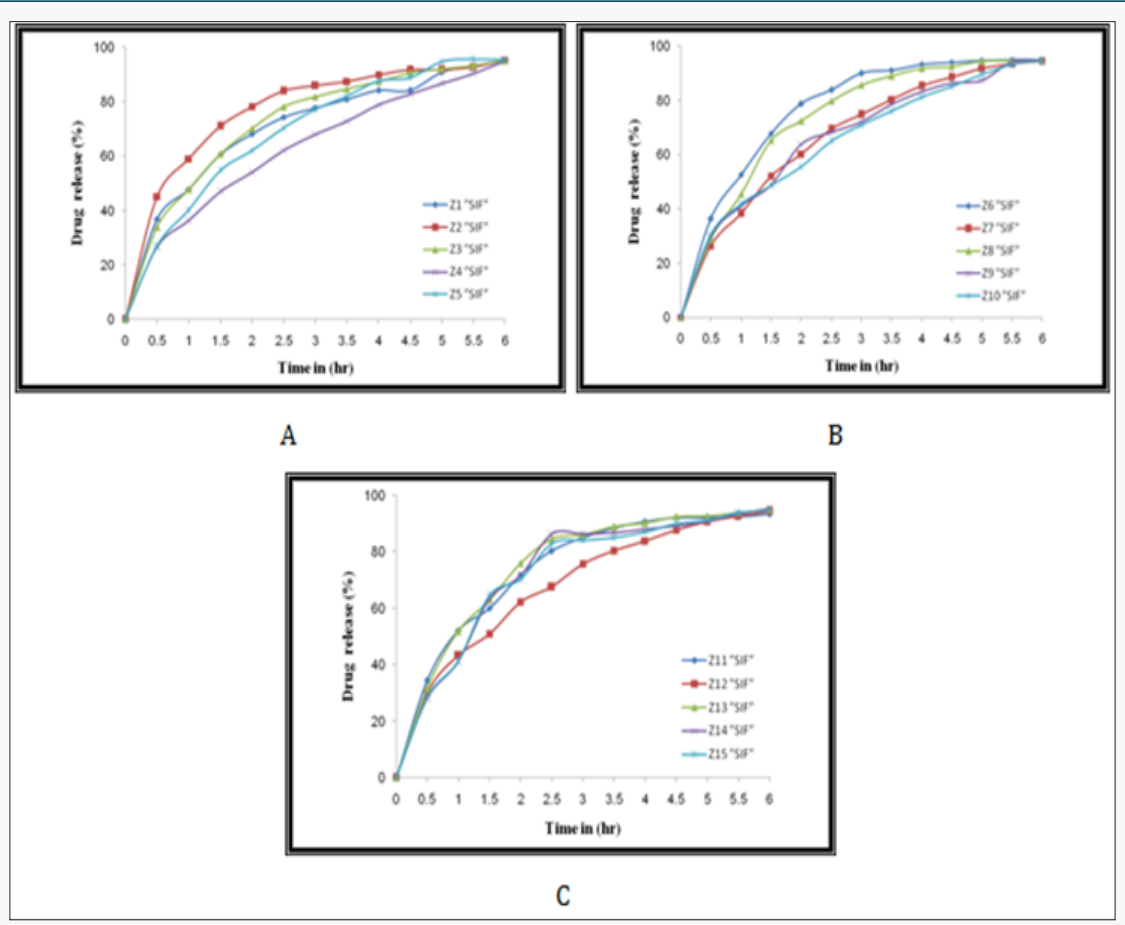

Figure 8: The \%Release of Gabapentin from: A) Z1-Z5, B) Z6-Z10 and C) Z11-Z15, in SGF.

The Higuchi release equation, which is one of the simplest mathematical equations, was used to determine the ratio of the absolute amount of the drug as a function of time based on the practical results obtained from the study of the release of the Gabapentin from all of the prepared hydrogel semi-IPN in distilled water, SGF and SIF and the Higuchi constant was determined according to the following equation [27]:

$$
Q_{(t)}=K_{H} t_{1 / 2}
$$

$Q_{(t)}$ : the cumulative percentage of the drug released at time $t$.

$\mathrm{k}_{\mathrm{H}}$ : Higuchi dissolution constant. and

t: Time in hours.

(Figure 9) illustrates the results of the application of the Higuchi equation for the semi-IPNs Z1-Z5. (Table 2) shows the Kinetic parameters of the release curve showing best fit with higher correlation with the Higuchi's equation for Z15-Z1 in distilled water, SGF and SIF.

Whereas:

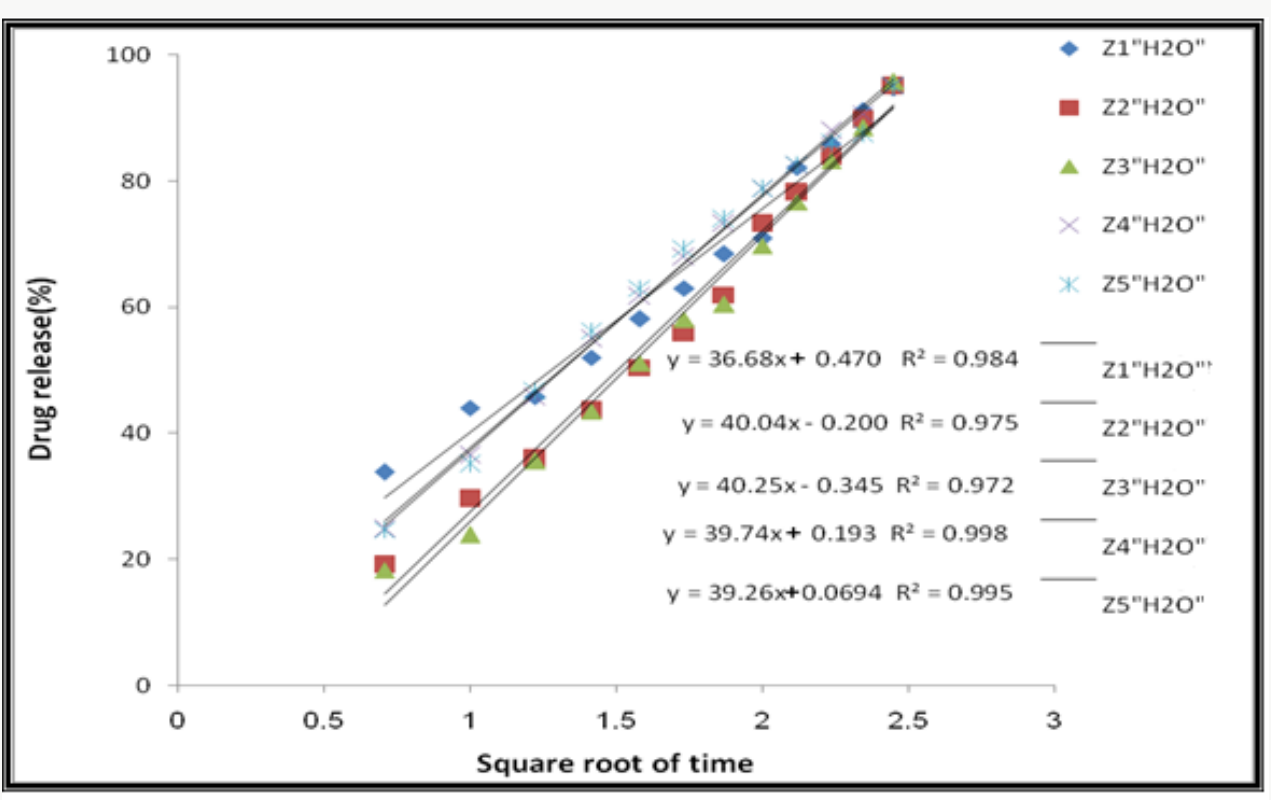

Figure 8: The \%Release of Gabapentin from: A) Z1-Z5, B) Z6-Z10 and C) Z11-Z15, in SGF. 
Table 2: Kinetic parameters of the release curve showing best fit with higher correlation with the Higuchi's equation for Z15-Z1 in distilled water, SGF and SIF.

\begin{tabular}{|c|c|c|c|c|c|c|}
\hline \multirow{2}{*}{ Sample } & \multicolumn{2}{|c|}{$\left(\mathbf{H}_{\mathbf{2}} \mathbf{0}\right)$} & \multicolumn{2}{c|}{ (SIF) } & \multicolumn{2}{c|}{ (SGF) } \\
\cline { 2 - 7 } & $\mathbf{R}^{2}$ & $\mathbf{R}^{\mathbf{2}}$ & $\mathbf{K H}$ & $\mathbf{R}^{\mathbf{2}}$ & $\mathbf{K H}$ & $\mathbf{R}^{\mathbf{2}}$ \\
\hline Z1 & 36.68 & 0.984 & 36.04 & 0.929 & 37.27 & 0.968 \\
\hline Z2 & 40.04 & 0.975 & 36.66 & 0.991 & 35.66 & 0.896 \\
\hline Z3 & 40.25 & 0.972 & 34.97 & 0.973 & 38.80 & 0.959 \\
\hline Z4 & 39.74 & 0.998 & 36.27 & 0.986 & 39.20 & 0.998 \\
\hline Z5 & 39.26 & 0.995 & 36.92 & 0.988 & 41.39 & 0.986 \\
\hline Z6 & 40.28 & 0.994 & 37.16 & 0.995 & 38.99 & 0.915 \\
\hline Z7 & 40.02 & 0.992 & 34.86 & 0.962 & 40.87 & 0.988 \\
\hline Z8 & 40.81 & 0.980 & 34.86 & 0.962 & 40.52 & 0.939 \\
\hline Z9 & 40.62 & 0.988 & 35.54 & 0.982 & 39.33 & 0.989 \\
\hline Z10 & 39.93 & 0.996 & 35.22 & 0.927 & 39.21 & 0.997 \\
\hline Z11 & 40.01 & 0.995 & 36.44 & 0.989 & 36.61 & 0.938 \\
\hline Z12 & 39.44 & 0.991 & 36.04 & 0.983 & 39.53 & 0.989 \\
\hline Z13 & 40.30 & 0.989 & 36.85 & 0.991 & 39.24 & 0.926 \\
\hline Z14 & 38.93 & 0.995 & 37.28 & 0.985 & 40.21 & 0.923 \\
\hline Z15 & 40.64 & 0.982 & 37.00 & 0.984 & 39.92 & 0.936 \\
\hline
\end{tabular}

\section{Conclusion}

The interaction between electrolytic polymers did not form a gel due to the unequal charge density, ionic strength and molecular weight of the electrolyte polymers which used in the preparation. The quaternary ammonium groups of copolymers and carboxylic groups in sodium alginate were found to enhance the change in the swelling ratio in distilled water, SGF and SIF. The SEM micrograph for swollen models showed the presence of large pores that increased the speed of release of the drug to environment.

\section{References}

1. W Tiyaboonchai (2003) Chitosan Nanoparticles: A Promising System for Drug Delivery. Naresuan University Journal 11(3): 51-66.

2. T Allen, P Cullis (2004) Drug delivery systems: Entering the mainstream. Science 303(5665): 1818-1822.

3. M Grassi, G Grassi (2005) Mathematical Modelling and Controlled Drug Delivery: Matrix System. Current Drug Delivery 2(1): 97-116.

4. D Breimer (1999) Future challenges for drug delivery. J Control Rele 62(1-2): 3-6.

5. A Singh (2000) The Use of Controlled Release Technology in Drug Delivery. J Pharm Sci 2.

6. R Verma, S Garg (2001) Current Status of Drug Delivery Technologies and Future Directions. Pharm Tech On Line 25(2): 1-14.

7. A Srinatha, J Pandit, S Singh (2008) Ionic crossed-linked chitosan beads for extended release of ciprofloxacin: in vitro characterization. Ind J Pharm Sci 70(1): 16-21.
8. M Zhang, T Tarn, N Xi (2004) Micro-/Nano-Devices for Controlled Drug Delivery, Robotics and Automation. IEEE Transactions on Control Systems Technology 2: 2068-2073.

9. M Aulton (2002) Pharmaceutics The Science of Dosage form design. ( $2^{\text {nd }}$ edn.), Churchil Livingstone London, UK 290-291.

10. J Park, fMnYe, Kf Park (2005) Biodegradable of Polymers for Microencapsulation of Drugs. Molecules 10(1): 146-161.

11. A Dias, M Hendriks (2009) Biomedical examine degradable polymers and their growing significance in controlled drug delivery. Samedan Ltd. Pharmaceutical Publishers 1: 16-22.

12. Y Perrie, T Rades (2010) Pharmacuetics: Drug Delivery and Targeting ( $1^{\text {st }}$ edn.), Published by the Pharmaceutical press, London, UK.

13.C Chiang (1995) Novel drug delivery systems for treatment of drug abuse. Nat Inst Hea guide 149: 21-39.

14. C Isha, S Nimrata, A Rana, G Surbhi (2012) Oral Sustained Release Drug Delivery System: An Overview. Inte Rese J Pharm 3(5): 56-62.

15. P Wong, D Edgren, L Dong, V Ferrari (1997) Banded prolonged release active agent dosage form. USP, 5667804.

16. S Rasheed, M Arief, P Vani, S Gajavalli, R Kishore (2010) Formulation and Evaluation of Sustained Release tablets of bupropiondhydrochloride. Rese J Pharm Bio Chem Sci 1(4): 1017-1025.

17. Md Schäfer Korting (2010) "Handbook of Experimental of Pharmacology" Springer Verlag Berlin Heidelberg 87-79.

18. A Bhargava, R Rathore, Y Tanwar, S Gupta, G Bhaduka (2013) Oral Sustained Release Dosage Form: An Opportunity to Prolong Release of Drug. Inter J Adv Rese Pharm Bio Sci 3(1): 7-14.

19. (2005) European Pharmacopoeia, $5^{\text {th }}$ Ed. council of Europe, Strasbourg.

20. C Nogueira, L Cabral, T Santos, A Marucci, F Alhaique (2003) Evaluation of New Polysaccharides Networks for extended-release purposes: mesquite seed gum (MSG), xanthan gum and chitosan. Braz J Pharm Sci 39(3): 274-288.

21. R Kulkarni, R Boppana, G Mohan, S Mutalik, N Kalyane (2012) $\mathrm{pH}-$ Responsive Interpenetrating Network Hydrogel Beads of Poly(acrylamide)-g-Carrageenan and Sodium alginate for Intestinal Targeted Drug Delivery: Synthesis, In vitro and In vivo Evaluation. J Colloid Interface Sci 367(1): 509-517.

22. S Dakhara, C Anajwala (2010) Polyelectrolyte Complex: A Pharmaceutical Review. Syst Rev Pharm 1(2): 121-127.

23. A Dobrynin, M Rubinstein (2005) Theory of Polyelectrolyte in Solutions and at Surfaces. Progress in Polymer Science 30(11): 1049-1118.

24. J Chen, M Liu, H Liu, L Ma, C Gao, et al. (2010) Synthesis and properties of thermo- and $\mathrm{pH}$-sensitive poly (diallyldimethylammonium chloride)/ poly (N,N-diethylacrylamide) semi-IPN hydrogel. Chem Eng J 159(1-3): 247-256.

25. D Kim, K Park (2004) Swelling and Mechanical Properties of Superporous Hydrogels of Poly(acrylamide-co-acrylic acid)/polyethylenimine interpenetrating polymer networks. Polymer 45(1): 189-196.

26. M Dinu, M Perju, E Drăgan (2011) Composite IPN ionic hydrogels based on polyacrylamide and dextran sulphate. Reactive and Functional Polymers 71(8): 881-890.

27. Ramteke KH, Dighe PA, Kharat AR, Patil SV (2014) Mathematical Models of Drug Dissolution: A Review. Sch Acad J Pharm 3(5): 388-396. 
(c) Co This work is licensed under Creative

Submission Link:

Submit Article

DOI: 10.32474/DDIPIJ.2018.01.000108

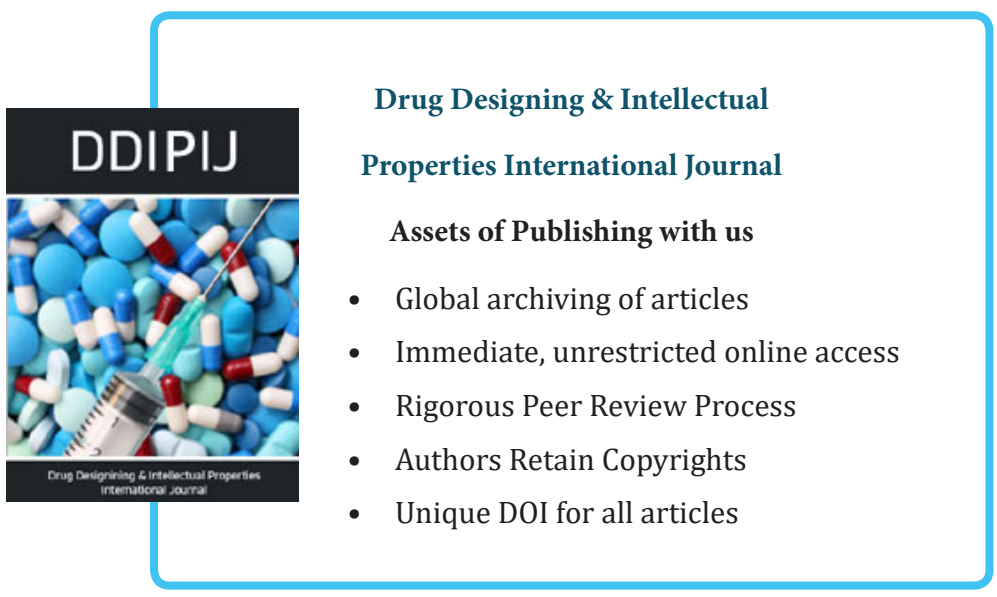

